\title{
Wireless Power Transfer via Magnetic Resonant Coupling by using Printed Circular Coil
}

\author{
Chia Chao Kang, Fatin Ayuni MS, Chia Yang Kang
}

\begin{abstract}
Nowadays, wiring connections are slowly fade out and replace with wireless connection due to the inconvenient of installing wiring connection in the wall or exposes to outside. Lately, the demand of wireless charging mobile phone or even charging by wireless in the car is attracted attention and the statistic shows that it is increasing rapidly. These paper proposed by using planar type spiral coil structure method to transfer the electrical signal energy to receiver part so as to act as wireless charging. It represented by 2 parallel FR4 printed circuit board that with the layout of planar spiral coil mutually coupled together in the free space. Analysis of electromagnetic transfer between both spiral coil and the resonant frequency was compute by coupled inductance theory. Simulations by using Advanced Design Systems based on coupling theory shows that when the air gap distance is changing, it will affecting the electromagnetic coupling efficiency of the wireless electromagnetic charging.
\end{abstract}

Index Terms: Magnetic Resonant coupling; Planar Spiral Coil; Wireless Power Transfer

\section{INTRODUCTION}

In recent years, mostly electronic devices that using wireless power transfer has been attracted many researcher and last but not least of $5 \mathrm{G}$ connection. The invention of wireless power transfer is to get rid of troublesome lines cable, power cord that normally applied in different type of applications such as charging electronic device [1] such as, tablets, mobile phones, EV car charging, portable laptop and others [2] in our daily life. Besides that, when there is a wireless techniques, it will constantly provide power to the device in any places that have the connectivity and it will help in terms of reducing the battery sizes. Thus, the product price will be reduce and the portability of the device will be increasing. Lastly, the implantable medical devices that used in the hospital will be power and recharge more easily through wireless power transfer.

The main principle of this wireless technique is to generate the resonant system that using two similar planar spiral coil with a separation distance between them. This is because the electromagnetic energy will be exchanged in both planar spiral coil with high frequency magnetic coupling and high efficiency level.As a results, the far field component of electromagnetic waves will be used to transfer the power between the transmitter part and receiver part.

Revised Manuscript Received on December 05, 2019.

Chia Chao Kang, Facilities Maintenance Engineering, Universiti Kuala Lumpur (MITEC), Bandar Seri Alam, Malaysia.

Fatin Ayuni MS, Facilities Maintenance Engineering, Universiti Kuala Lumpur (MITEC), Bandar Seri Alam, Malaysia.

Chia Yang Kang, Faculty of Electrical Engineering, Swinburne University, Kuching Sarawak, Malaysia.
In this paper, different transmission distance between the two planar spiral coil structures is presented. The parameters of the planar spiral coil are simulated and computed using analytical method and finite element method (FEM) in advanced design system. Lastly, the design of the planar spiral coil model is used to evaluate the effect of electromagnetic coupling.

\section{EQUIVALENT CIRCUIT OF MAGNETIC COUPLING}

\section{A. Equivalent Lumped Element of Single Spiral Coil}

In this section, the basic characteristic of the planar spiral inductor and its relationship to the wireless power transfer is discussed. The parameter of the planar spiral inductor can predict the behavior of the system. Based on wireless power transfer system, a circular planar inductor is chosen to form the basic prototype rather than the square spiral inductor because of the corner resistance can be eliminated. A mathematical model is established, as in Fig. 1 below to describe and understand the effect on the resonance frequency and inductance value.

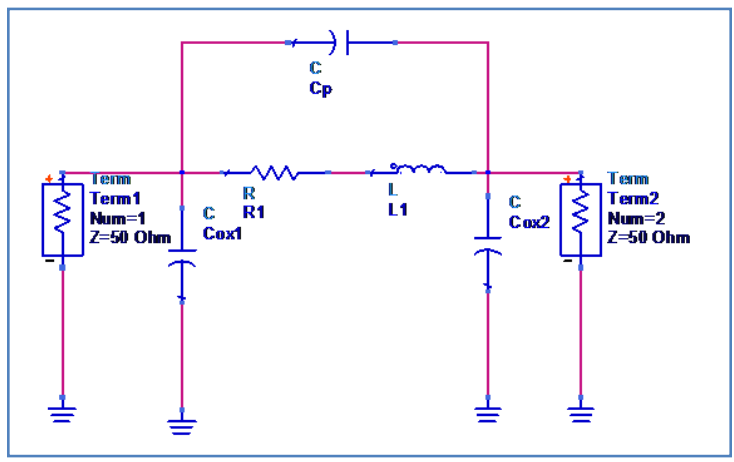

Fig. 1 Circuit model of single spiral coil

The model consists of crossover capacitance $\mathrm{Cp}$, series resistance $\mathrm{R}$ and inductance $\mathrm{L}$, capacitance $\operatorname{Cox} 1$ and Cox 2 between the spiral track and ground. The inductance of the circular spiral inductor is computed using the Wheeler formula [3].

$$
L \cong \frac{\mu_{\circ} n^{2} d_{a v g} c_{1}}{2}\left(\ln \left(\frac{c^{2}}{\rho}\right)+c_{3} \rho+c_{4} p^{2}\right)
$$

Where $c_{1}, c_{2}, c_{3}, c_{4}$ are coefficient, $d_{\text {out }}=$ outer diameter, $d_{\text {in }}$ $=$ inner diameter, $\mathrm{p}$ is defined as fill ratio $\rho=\left(d_{\text {out }}-d_{\text {in }}\right) /\left(d_{\text {out }}+d_{\text {in }}\right)$ an $n$ is the number of turns and $\mu_{0}$ is the permeability of free space. 


\section{B. Analysis of Coupled Magnetic Resonance}

The fundamental theory of magnetic resonant coupling [4] can be represented by LC resonance. Thus, magnetic coupling that are using equivalent circuit for power transfer system using as in Fig. 2 will be discussed. Default characteristic of $50 \mathrm{ohm}$ impedance is used in this system. When voltage is applied to the transmitting coil, the magnetic field will be created, current will be induced in the receiving spiral inductor due to electromagnetic forces. It will create wireless power transfer that electric energy will flow and does not need conductive wires.

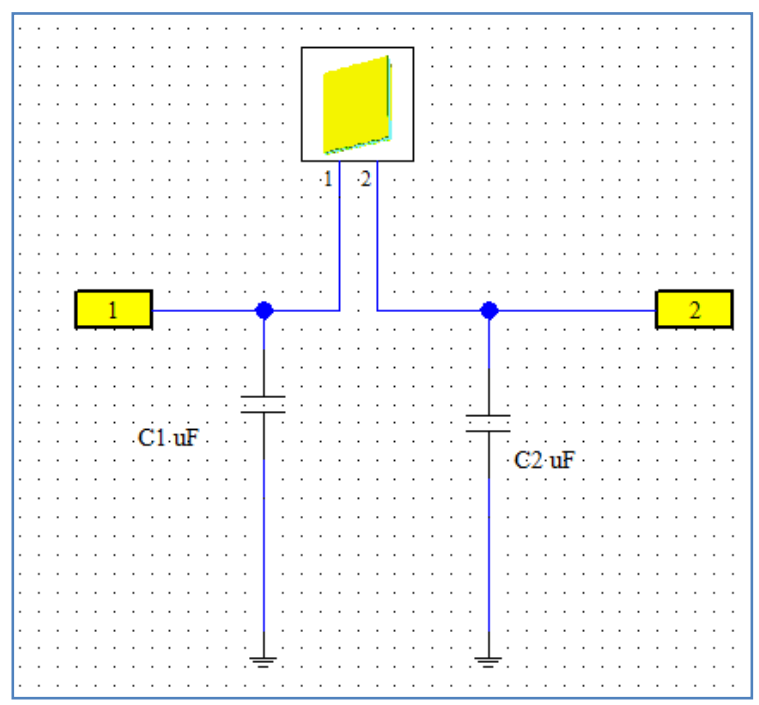

Fig. 2 Magnetic resonant coupling circuit

The transmitter coil is ideally connected to the sinusoidal voltage source and the receiver coil is connected to a load. In Fig. 2, $C_{1}$ and $C_{2}$ is connected in parallel to create the resonance circuit, their values are,

$$
\begin{aligned}
& C_{1}=\frac{1}{(2 \pi f)^{2} L_{1}} \\
& C_{2}=\frac{1}{(2 \pi f)^{2} L_{2}}
\end{aligned}
$$

Equation (4) represents the basic resonant frequency equation of wireless power transfer system.

$$
f=\frac{1}{2 \pi \sqrt{L C}}
$$

Besides that, when the frequency is increased, the current is concentrated to flow near the surface of the spiral inductor and it will cause the skin effect. The skin depth can be defined as

$$
\delta=\sqrt{\rho /(\pi} \mu f)
$$

Magnetic coupling is generated between the transmitter and receiver planar spiral coil in the direction of vertically oriented as Fig. 3.

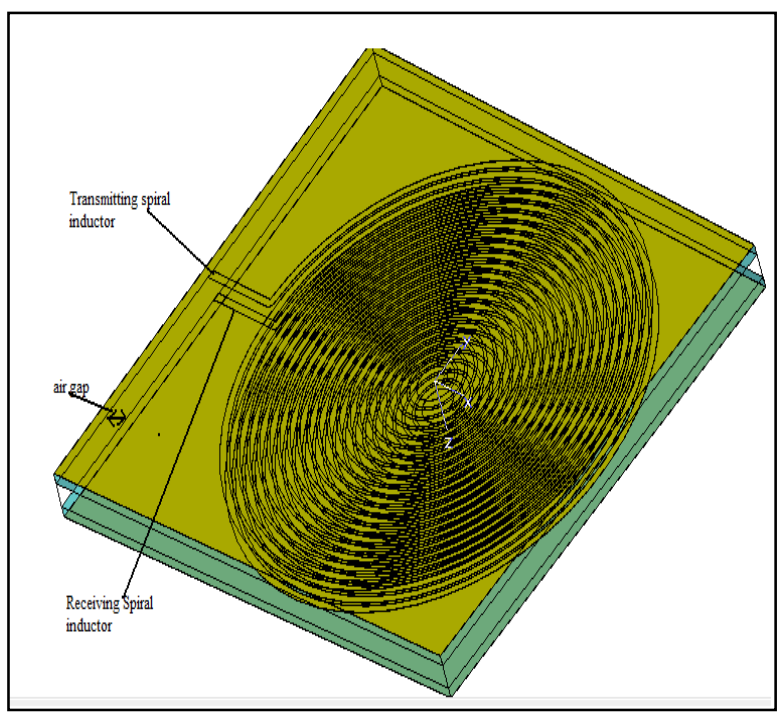

Fig. 3 Parallel spiral coil with air gap

Distance between two planar spiral coil are closely related to factors such as distance of transmission. Thus, as the distance changes, the impedance will be varied, therefore it will affect how well the spiral inductor is coupled to each other [6].

\section{PERFORMANCE OF EVALUATION}

When the source of alternating current power supply is applied through the planar spiral coil, it will generate constantly varying magnetic field around the primary coil, the magnetic field, in turn will induces voltages in the secondary spiral inductor. The magnetic resonance coupling is evaluated based on the varying distance between the primary and secondary spiral inductor. Figure 2 set up shows planar spiral inductor placed with a $1 \mathrm{~mm}$ distance. It can be observed that the transfer coefficient $S_{21}$ at $922.4 \mathrm{MHz}$ is $-3.92 \mathrm{~dB}$ as shown in Fig. 4.

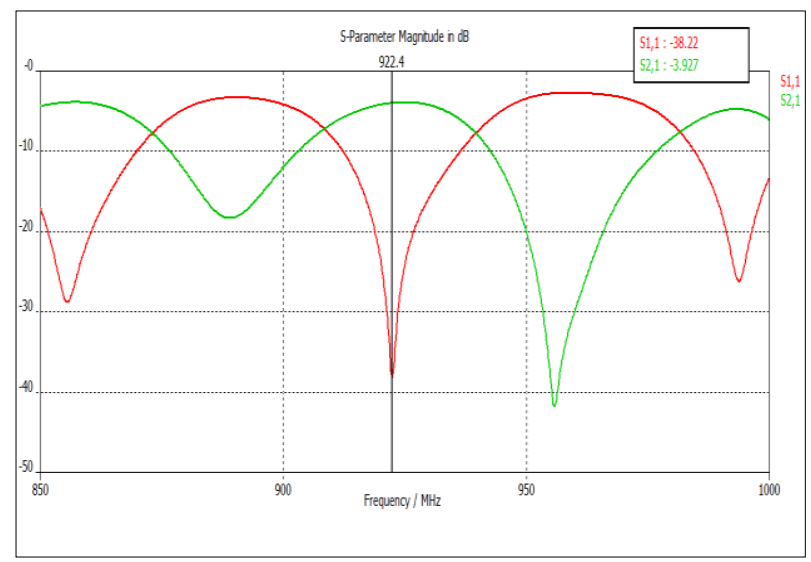

Fig. 4 Distance separation of $S_{21}$ is $1 \mathrm{~mm}$

When the turn of the spiral is unchanged, but the distance separation became $1.3 \mathrm{~mm}$. It can be observed that $S_{21}$ at 922.4MHz is $-5.16 \mathrm{~dB}$ as Fig. 5. As the air gap between the spiral inductor increase, the coupling in between the spiral inductor became weaken, thus it will affect the transfer coefficient to be decreasing. 


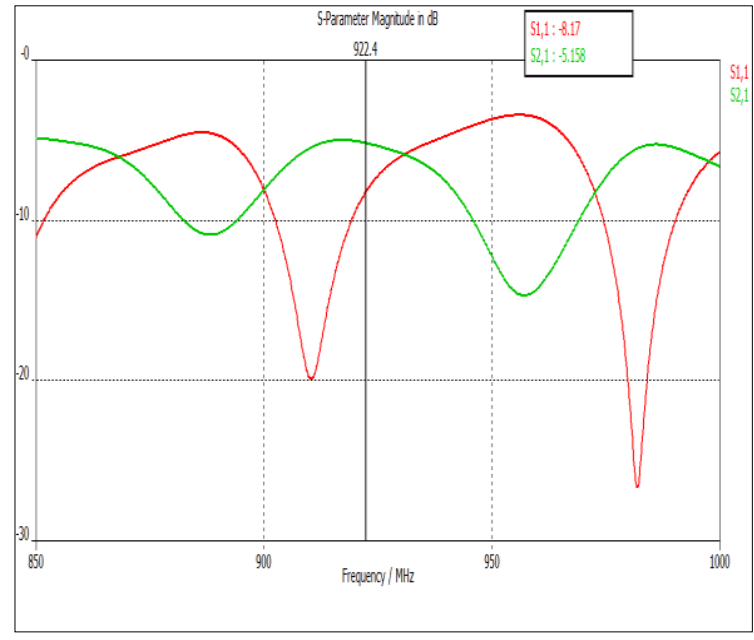

Fig. 5 Distance separation of $S_{21}$ is $1.3 \mathrm{~mm}$

When distance separation is increased, the transfer coefficient has dropped to around $1.2 \mathrm{~dB}$. Next, the distance is further increased until $1.9 \mathrm{~mm}$ and observation on $S_{21}$ is shown in Fig. 6.

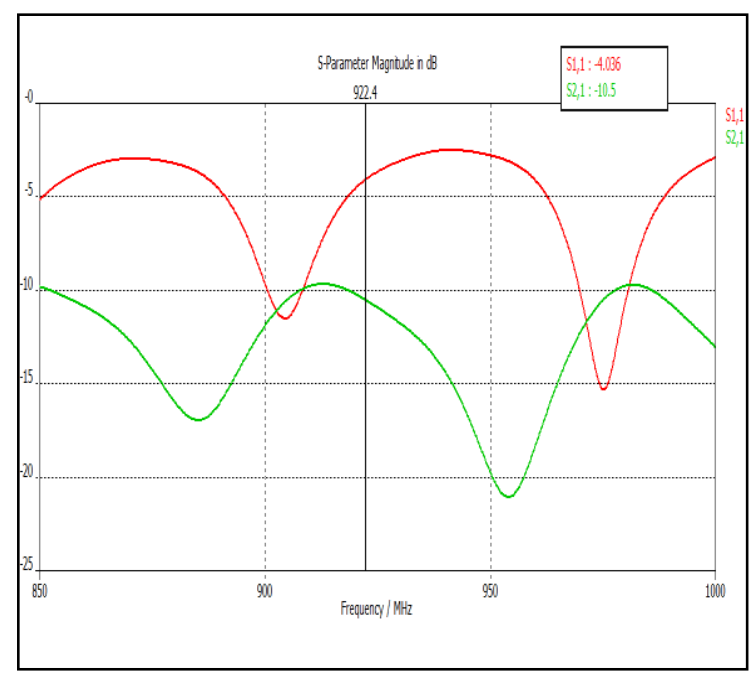

Fig. 6 Distance separation of $S_{21}$ is $1.9 \mathrm{~mm}$

As the separation distance is $1 \mathrm{~mm}, \mathrm{~S}_{21}$ of $-10.5 \mathrm{~dB}$ at $922.4 \mathrm{MHz}$ has dropped about $6.57 \mathrm{~dB}$. As an overall observation, distance is inversely proportional to transfer coefficient. This is due to fact that when the separation of space is becoming bigger, the maximum value of electromagnetic field is not properly delivered to the receiver side of spiral coil and there will be power loss.

\section{CONCLUSION}

Use The paper presented multi turn magnetic resonance coupling of wireless power transfer system for planar spiral coil on FR4 board is shown. The magnetic resonant coupling is evaluated based on the equivalent circuit system and electromagnetic analysis in order to observe the results. When the distances increase, the energy deliver from the transmitter side to the receiver will be decrease.This is because attenuation nature and spreading of radioactive power in space, last but not least it requires line of sign. Based on the results, further research will extensively carry out to produce more meaningful energy transfer using multi turn circular spiral inductor base on magnetic coupling resonance.

\section{ACKNOWLEDGMENT}

The authors gratefully acknowledge that this work was supported by the Universiti Kuala Lumpur (MITEC)

\section{REFERENCES}

1. Hema Ramachandran, Dr. Bindu G.R, "Wireless Charging of Lighting Gadgets Using Low Q Resonant Coupling," Proceedings of the International Conference on Advances in Computing, Communications and Informatics, pp. 275-278, 2012.

2. B.J. Jang, S. Lee, H. Yoon, "HF-Band Wireless Power Transfer System: Concept, Issues and Design," Progress in Electromagnetics Research, vol. 124, pp. 211-231, 2012.

3. Uei-Ming Jow, Maysam Ghovanloo, "Design and Optimization of Printed Spiral Coils for Efficient Transcutaneous Inductive Power Transmission," IEEE Transcations on Biomedical Circuits and System, Vol. 1, No. 3, 2007.

4. Takehiro Imura, Toshiyuki Uchida, Yoichi Hori, "Flexibility of Contactless Power Transfer using Magnetic Resonance Coupling to Air Gap and Misalignment for EV. World Electric Vehicle Journal," Vol. 3, 2009.

5. Inder Bahl, Lumped Elements for RF and Microwave Circuits, Artech House, 2003.

6. J. Choi, C. Seo, "Analysis on Transmission Efficiency of Wireless Energy Transmission Resonator Based on Magnetic Resonance," Progress in Electromagnetics Research M. Vol. 19, pp. 221-237, 2011.

7. Hussain, A., Manikanthan, S.V., Padmapriya, T. Nagalingam M. Wireless Networks (2019) https://doi.org/10.1007/s11276-019-02121-4 Genetic algorithm based adaptive offloading for improving IoT device communication efficiency.

8. Manikanthan, S.V., Padmapriya, T., An efficient cluster head selection and routing in mobile WSN, International Journal of Interactive Mobile Technologies, 2019.

\section{AUTHORS PROFILE}

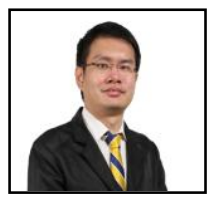

DR KANG CHIA CHAO is currently a senior lecturer (FaMESection) at Malaysian Institute of Industrial Technology (MITEC), Universiti Kuala Lumpur (UniKL). He is a registered Graduate Engineer under Board of Engineering Malaysia (BEM), member of Malaysian Society of Engineering Technology (MySET), member of Malaysia Board of Technologists and member of Institute of Electrical and Electronic Engineering and Technology (IEEE). He received his Bachelor of Electrical \& Electronic Engineering at Northumbria University in Newcastle upon Tyne, Master of Science in Electronic System Design Engineering and Doctor of Philosophy in Microwave and System Satellite at UniversitiSains Malaysia. He has several years of industrial working experience in electrical and electronic field of engineering as a product engineer prior joining as academician. From 2016 until now, he also is appointed as a section coordinator for INTRA. Besides that, he is also a professional trainer certified by HRDF Malaysia, involved in First Aid training \& Emergency Response Team and a certified thermography level (1). 
FATIN AYUNI BINTI MOHD SUHAIMI is currently a senior lecturer (FaME Section) at Malaysian Institute of Industrial Technology (MITEC), Universiti Kuala Lumpur (UniKL). He is a registered Graduate Engineer under Board of Engineering Malaysia (BEM). She completed her Master from University of Wollongong, New South Wales. She received her Bachelor ofEngineering (Hons) in Mineral Resources Engineering in Universiti Sains Malaysia.

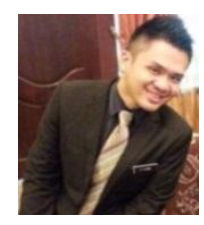

KANG CHIA YANG is currently a lecturer from Electrical Engineering Department, Mukah Polytechnics. He received his Bachelor of Electrical System Engineering at University Malaysia Perlis. He is involve in the VHDL implementation of a multiplier generator with the development of QUARTUS Electronic Design Automation (EDA) and FPGA System on programmable chip (SOPC) during his study

at Aalen University, Germany. He has several years of working experience as a Test Engineer in AIC Semiconductor Sdn.Bhd and also FUJI Electric before becoming an academician. 